\title{
On Fuzzy Sequences, Fixed Points and Periodicity in Iterated Fuzzy Maps
}

\author{
H.N. Teodorescu
}

\author{
Horia Nicolai Teodorescu \\ Gheorghe Asachi Technical University, \\ Iasi, Romania \\ Institute for Computer Science, \\ Romanian Academy, Iasi Branch \\ E-mail: hteodor@etc.tuiasi.ro
}

\begin{abstract}
I exemplify various elementary cases of fuzzy sequences and results related to the iteration of fuzzy mappings and to fuzzy logic systems (FLS). Several types of fuzzy logic system iterations are exemplified in relationship with oscillations in FLS and with the problem of stability in fuzzy logic control. I establish several conditions for fixed points and periodicity of the iterations based on fuzzy systems.
\end{abstract}

Keywords: modeling, control, fuzzy map iteration, fuzzy sequence, behavior.

\section{Introduction}

In this paper, I provide simple examples of iteration of fuzzy sets and I discuss the asymptotic behavior of their successive iterations. I discuss several cases of iterated fuzzy logic and arithmetic systems with and without defuzzification and determine conditions for fuzzy sets that are fixed points. The topic was dealt with in the literature in the early approaches by Kloeden [1] and in [2]- [9], but attracted little interest during the last two decades. However, sequences of fuzzy numbers and various generalizations constituted recently the subject of a large number of papers [10]- [21], while many applications indirectly referred to fuzzy sequences [22]- [24]. However, few recent papers, like [24], addressed fuzzy sequences and the problem of fixed points in fuzzy mappings with relation to fuzzy systems and control.

In this paper, a fuzzy map is any application from a set of fuzzy sets to itself. A fuzzy system is a function with fuzzy set-valued variables, also named inputs, and fuzzy set values, named output. An iterated fuzzy system can be seen as a fuzzy system, in the sense used in control, but without defuzzification, that has a connection from the fuzzy output to the fuzzy input through a unitary delay loop. Analyzing fuzzy iterations has therefore applications in control, in decision making, and in modeling, among others.

The connection between iterations and sequences is straightforward: while iterations are defined by $f^{[n+1]}=f\left(f^{[n]}\right)$, many sequences are obtained as $a_{n+1}=f\left(a_{n}\right), a_{n+1}=f\left(a_{n-1}, a_{n}\right)$, $a_{n+1}=f\left(g\left(a_{n-1}\right), a_{n}\right)$, or by similar formulas.

A fixed point of a specified fuzzy function (system) iteration is a fuzzy set, $\tilde{A}$, (membership function, $\mu_{\tilde{A}}$ ) that is preserved under the iteration; in other words, when presented to the input of the fuzzy system, it is found unchanged at its output.A periodical fuzzy system will recover after a fixed number of iterations - the period - the initial input membership function as its output. Assume that a metric space has been defined on a space of fuzzy sets. Subsequently, when convergence of sequences is considered in the respective metric space, I mean that $\tilde{A}_{n} \rightarrow \tilde{A}_{\infty}$ iff $d\left(\tilde{A}_{n}, \tilde{A}_{\infty}\right) \longrightarrow^{n \rightarrow \infty} 0$. Whenever absolute convergence will be meant, the following definition applies: $\tilde{A}_{n} \rightarrow \tilde{A}_{\infty}$ iff $\left|\mu_{\tilde{A}_{n}}(x)-\mu_{\tilde{A}_{\infty}}\right| \longrightarrow^{n \rightarrow \infty} 0 \forall x$. In this paper, the primary interest is in absolute convergence. 
A basin of attraction of a specified input fuzzy set, which is a fixed point for a specified fuzzy system, is the set of membership functions that determine the output of the fuzzy system to tend during the iteration to the specified fuzzy set. The applications of the above concepts in the real space are well known to control engineers, biologists, and virtually in all disciplines.

I denote by $[0,1]^{R}$ the set of functions from $\mathrm{R}$ to $[0,1]$. Membership functions used at the input and at the output of the fuzzy logic systems belong to $[0,1]^{R}$. Being given an application $\varphi:[0,1]^{R} \rightarrow[0,1]^{R}$, the successive iteration of $\varphi$ with initial condition $\tilde{A}_{0}$ is $\tilde{A}_{0} \rightarrow \tilde{A}_{1}=$ $\varphi\left(\tilde{A}_{0}\right), \tilde{A}_{2}=\varphi\left(\tilde{A}_{1}\right)=\varphi^{[2]}\left(\tilde{A}_{0}\right), \tilde{A}_{3}=\varphi\left(\tilde{A}_{2}\right)=\varphi^{[3]}\left(\tilde{A}_{0}\right), \ldots$.

A fuzzy logic system (FLS) is basically an application $\varphi:[0,1]^{R} \rightarrow[0,1]^{R}$, which is defined in a specific manner, using rules. When a discrete-time feedback loop is added to a fuzzy logic system, the equivalent operation is an iteration of the function $\varphi$ starting with an initial input membership function $\tilde{A}_{0}$. The operation of the loop is expressed by the successive iterations $\varphi^{[n]}\left(\tilde{A}_{0}\right), \mathrm{n}=1,2,3, \ldots$. From the application point of view, an iteration of a FLS can be seen as a FLS with a discrete time, constant loop, like in Fig.1.

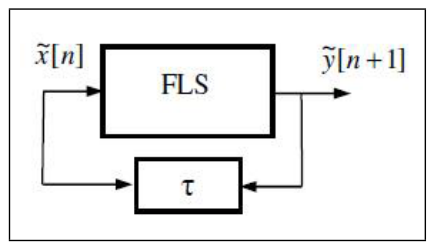

Figure 1: Iteration of a FLS represented as a FLS with a delay loop. The loop "gain" is unitary. The operation is in discrete time.

The set $[0,1]^{R}$, when endowed with one of the usual definitions of distance between functions, becomes a metric space and has the corresponding induced topologic structure. Therefore, we are able to talk about limit points in that topologic space and about the asymptotic behavior of the successive iteration of an application represented by a FLS. When a metric is needed, I use $d(\tilde{A}, \tilde{B})=\int_{-\infty}^{\infty}\left(\mu_{\tilde{A}}(x)-\mu_{\tilde{B}}(x)\right)^{2} d x$, where $\tilde{A}, \tilde{B}$ stand for the output of the FLSs, assuming integrability.

In the next section, which is preparatory, I exemplify various ways for building sequences of fuzzy sets. In section 3, I discuss sequences generated by FLSs. Conclusions are derived in Section 4 . Throughout this paper, $\diamond$ denotes the end of a proof, example, or a line of reasoning.

\section{Elementary examples of fuzzy sequences}

\subsection{Games based on fuzzy sequences produced by logic operations over fuzzy sets}

I start illustrating the topic of fuzzy sequence by a simple example based on logic operations with fuzzy sets. Consider the following game: Three school colleagues have a high spirit of contradiction. When they start a discussion, they typically start with two disparate opinions; then, each of them is ready to contradict what the last speaker told and partly agree and partly disagree with the ante-precedent speaker; to do so, they answer with what at the same time contradicts the predecessor, yet agrees with the ante-predecessor. They take turns in expressing their opinions in that way. Can they ever come to a conclusion? If yes, after how many steps of mutual contradictions? (I would say that the game is not far from what seldom happens in real life, so this example is not devoid of interest. The problem is if a group of three skillful contradictors still can agree under fuzzy logic.) 
This "game" is not possible in binary logic, but makes sense in the frame of fuzzy logic. Consider two fuzzy sets, $\tilde{A}_{0}$ and $\tilde{A}_{1}$ representing the first two opinions. The third colleague's and the subsequent responses define a sequence of fuzzy sets by $\tilde{A}_{n+1}=\neg \tilde{A}_{n} \cap \tilde{A}_{n-1}, n \geq 1$, where $\neg \tilde{A}_{n}$ denotes the complement of the fuzzy set $\tilde{A}_{n}$. The above recurrence, together with the initial conditions $\tilde{A}_{0}$ and $\tilde{A}_{1}$, defines the fuzzy set sequence. It is easy to see that the sequence is $\tilde{A}_{0}, \tilde{A}_{1}, \tilde{A}_{2}=\neg \tilde{A}_{1} \cap \tilde{A}_{0}, \tilde{A}_{3}=\neg \tilde{A}_{2} \cap \tilde{A}_{1}, \ldots$. The membership functions of $\tilde{A}_{3}$ is $\mu_{3}(x)=\min \left(\mu_{1}(x), 1-\mu_{2}(x)\right)$, or $\mu_{3}(x)=\min \left(\mu_{1}(x), 1-\min \left(\mu_{0}(x), 1-\mu_{1}(x)\right)\right)$.

(i) Assume $\mu_{0}(x)<1-\mu_{1}(x)$. Then $\mu_{3}(x)=\min \left(\mu_{1}(x), 1-\mu_{0}(x)\right)$. Because $\mu_{0}(x)<1-\mu_{1}(x)$, $\mu_{1}(x)<1-\mu_{0}(x)$, thus $\mu_{3}(x)=\min \left(\mu_{1}(x), 1-\mu_{0}(x)\right)=\mu_{1}(x)$.

(ii) Assume $\mu_{0}(x) \geq 1-\mu_{1}(x)$. Then $\mu_{3}(x)=\min \left(\mu_{1}(x), 1-\left(1-\mu_{1}(x)\right)\right)=\mu_{1}(x)$. Therefore, $\tilde{A}_{3}=\tilde{A}_{1}$. Next, $\tilde{A}_{4}=\neg \tilde{A}_{3} \cap \tilde{A}_{2}=\neg \tilde{A}_{1} \cap \tilde{A}_{2}$ and $\mu_{4}(x)=\min \left(\mu_{2}(x), 1-\mu_{1}(x)\right)$, or $\mu_{4}(x)=$ $\min \left(\min \left(1-\mu_{1}(x) ; \mu_{0}(x)\right), 1-\mu_{1}(x)\right)$.

(i) Assume $\mu_{0}(x)<1-\mu_{1}(x)$. Then $\mu_{4}(x)=\min \left(\mu_{0}(x), 1-\mu_{1}(x)\right)=\mu_{0}(x)$.

(ii) Assume $\mu_{0}(x) \geq 1-\mu_{1}(x)$. Then $\mu_{4}(x)=\min \left(1-\mu_{1}(x), 1-\mu_{1}(x)\right)=1-\mu_{1}(x)$. Thus, $\tilde{A}_{4}=\neg \tilde{A}_{1}$. Next, $\tilde{A}_{5}=\neg \tilde{A}_{4} \cap \tilde{A}_{3}=\tilde{A}_{1} \cap \tilde{A}_{1}=\tilde{A}_{1}$.

If the response strategy is changed, that is $\tilde{A}_{n+1}=\tilde{A}_{n} \cap \neg \tilde{A}_{n-1}, n \geq 1$, the game is less simple. Indeed, the membership functions of $\tilde{A}_{2}$ and $\tilde{A}_{3}$ are $\mu_{2}=\min \left(\mu_{1}, 1-\mu_{0}\right), \mu_{3}=\min \left(\min \left(\mu_{1}, 1-\right.\right.$ $\left.\left.\mu_{0}\right), 1-\mu_{1}\right)$. Whenever $\mu_{1}(x)<1-\mu_{0}(x), \mu_{3}(x)=\min \left(\mu_{1}(x), 1-\mu_{1}(x)\right), \tilde{A}_{3}=\tilde{A}_{1} \cap \neg \tilde{A}_{1}$, else $\tilde{A}_{3}=\neg \tilde{A}_{0} \cap \neg \tilde{A}_{1}$. The next step is $\tilde{A}_{4}, \mu_{4}=\min \left(\mu_{3}, 1-\mu_{2}\right)$.

\subsection{Fuzzy sequences produced by arithmetic operations over values of the membership functions}

Arithmetic operations with fuzzy numbers have been used to build chaotic processes by Kloeden [1]. Here I exemplify how, based on sequences of fuzzy sets and operating them through logic operations and using arithmetic manipulations of the elements of the sequences, one can obtain new sequences with the desired properties. Consider the fuzzy set $\tilde{A}_{0}$ with

$$
\mu_{0}(x)= \begin{cases}x^{2} & x \in[0,1] \\ 0 & \text { elsewhere }\end{cases}
$$

and the sequence of singletons with the membership functions

$$
\mu_{n}(x)= \begin{cases}1 & x=\frac{1}{n} \\ 0 & \text { elsewhere }\end{cases}
$$

Define $\tilde{C}_{n}$ by $\tilde{C}_{0}=\tilde{A}_{0}$ and $\tilde{C}_{n}=\tilde{C}_{n-1} \cup \tilde{B}_{n}$ for $n>0$. The graph of the fuzzy set $\tilde{C}_{n}$ looks like in Figure 2, where, for convenience, I have drawn a line to show where a singleton occurs. Recall that a small full circle on the graph conventionally denotes the actual value at a point of discontinuity of a function, while a small empty circle denotes the lack of value on the graph of a continuous function. The number of singularities (discontinuities) of the membership function of the sets $\tilde{C}_{n}$ is equal to n. $\diamond$

Define a sequence of fuzzy sets, $\left\{\tilde{u}_{n}, n=1,2, \ldots\right\}$, based on the fuzzy numbers $\nu_{0}, \nu_{1}$,

$$
\begin{gathered}
\mu_{\tilde{\nu}_{0}}(x)=\left\{\begin{array}{cc}
0 & \text { if } x<0 \\
1-x / 3 & \text { or } x>3
\end{array},\right. \\
\mu_{\tilde{\nu}_{1}}(x)=\left\{\begin{array}{lll}
0 & \text { if } & x \neq 1 \\
1 & \text { else },
\end{array}\right.
\end{gathered}
$$




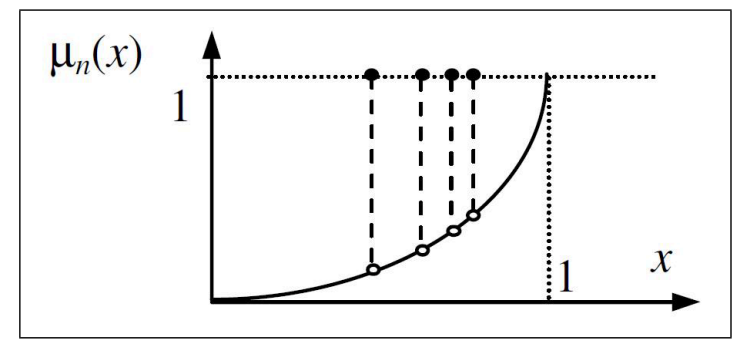

Figure 2: .

$$
\text { as } \quad \tilde{u}_{n}=\tilde{\nu}_{0} \cap \neg\left(3 \cdot \tilde{\nu}_{1}\right)^{n} \text {. }
$$

I propose to graphically and analytically find the first two sets in the series, then to write their expressions, determining if there is a limit of the sequence. According to the operations with fuzzy numbers in the framework of the fuzzy arithmetic,

$$
\mu_{\left(3-\nu_{1}\right)^{n}}(x)=\left\{\begin{array}{lll}
1 & \text { if } & x=3^{n} \\
0 & & \text { elsewhere. }
\end{array}\right.
$$

This expression is obtained by applying the definition of the product of fuzzy numbers:

$$
\tilde{m} \cdot \tilde{n}=\tilde{q}, \mu_{q}(w)=\max _{\text {all }} \min \left(\mu_{m}(y), \mu_{n}(z)\right) .
$$

For singletons $\tilde{\nu}$, the expression $\min \left(\mu_{m}(y), \mu_{n}(z)\right) \neq 0$ only if $z$ corresponds to the value of the singleton. From the defining relations, $\tilde{u}_{1}=\tilde{\nu}_{0} \cap \neg\left(3 \cdot \tilde{\nu}_{1}\right)^{1}$ and

$$
\begin{aligned}
& \mu_{3 \nu_{1}}(x)=\left\{\begin{array}{cc}
1 & \text { if } \quad x=3 \\
0 & \text { elsewhere }
\end{array}\right. \\
& \mu_{-3 \nu_{1}}(x)=\left\{\begin{array}{lll}
1 & \text { if } & x \neq 3 \\
0 & \text { if } & x=3,
\end{array}\right. \\
& \mu_{u_{1}}(x)=\left\{\begin{array}{l}
0 \quad \text { if } x<3 \text { or } x>3 \\
1-x / 3 \text { if } x \in[0,3] \mid\{3\} \\
0 \quad \text { if } x=3 .
\end{array}\right.
\end{aligned}
$$

Therefore, $\mu_{u_{1}}=\mu_{\nu_{0}}$. Similarly,

$$
\begin{gathered}
\tilde{u}_{2}=\tilde{\nu}_{0} \cap \neg\left(3 \cdot \tilde{\nu}_{1}\right)^{2}, \\
\mu_{u_{1}}(x)=\left\{\begin{array}{lll}
0 & \text { if } x<0 & \text { or } x>3 \\
1-x / 3 & \text { else }
\end{array}\right.
\end{gathered}
$$

Thus, $\mu_{u_{1}}=\mu_{\nu_{0}}$. Moreover, for all $n>2, \mu_{u_{n}}=\mu_{\nu_{0}}$ (see Figure 3). Therefore, $\tilde{\nu}_{0}$ is the limit of the constant sequence $\left\{\tilde{u}_{n}\right\}$.

Further sequences are obtained by varying the manipulation of the membership functions in the precedent sequence. For example, changing the definition condition $\tilde{u}_{n}=\tilde{\nu}_{0} \cap \neg\left(3 \cdot \tilde{\nu}_{1}\right)^{n}$ with the condition $\tilde{u}_{n}=\tilde{\nu}_{0} \cap \neg\left(\frac{2}{3} \cdot \tilde{\nu}_{1}\right)^{n}$, a new sequence is obtained. 


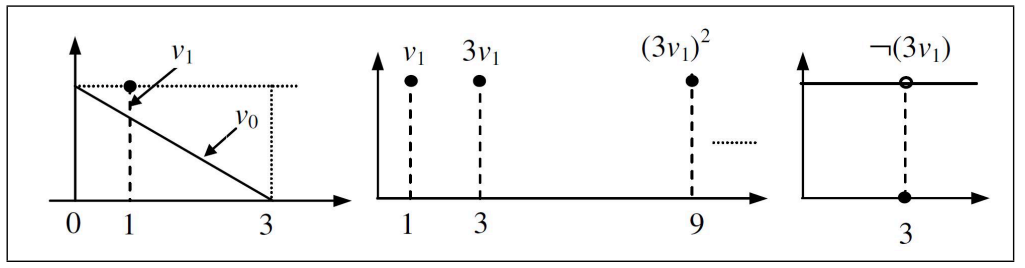

Figure 3: Explanatory graphs .

\subsection{Sequences defined by conditions on the membership functions}

Sequences of fuzzy sets can be conveniently defined based on sequences of real numbers standing for $\alpha$-cuts. Another fuzzy sequence with limit is exemplified subsequently. Consider a fuzzy set $\tilde{A}_{0}$ satisfying the condition $\mu_{0}(x)>0$ if $\mathrm{x} \in(0,1)$ and $\mu(x)=0$ elsewhere. I denoted $\mu_{\tilde{A}_{0}}$ by $\mu_{0}$. Apply the following iterative procedure to generate a sequence of fuzzy sets:

$$
\mu_{n}(x)= \begin{cases}\mu_{n-1}(x) & \text { if } \mu_{n-1}(x)<\left(\frac{2}{3}\right)^{n}, \quad n \geq 1, \\ 0 & \text { elsewhere, }\end{cases}
$$

where $\mu_{n}$ represents the membership function of the fuzzy set obtained at the $n$-th iteration. It is easy to check that $\mu_{n} \longrightarrow_{n \rightarrow \infty} \emptyset$ (see Figure 4.)

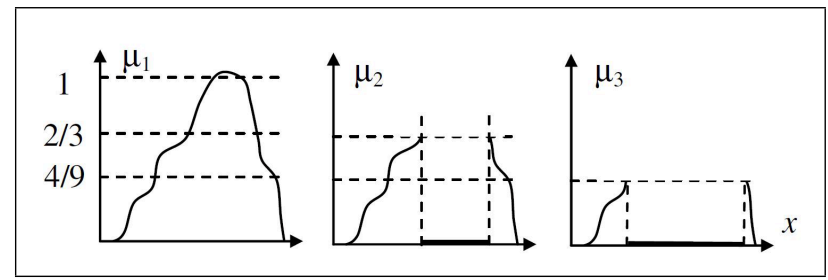

Figure 4: A sequence of fuzzy sets tending to the null fuzzy set, $\mu(x)=0 \forall \mathrm{x} \in \mathbf{R}$.

Periodical fuzzy sequences are defined as a direct extension of periodicity for real valued sequences. I define a sequence of fuzzy sets as follows:

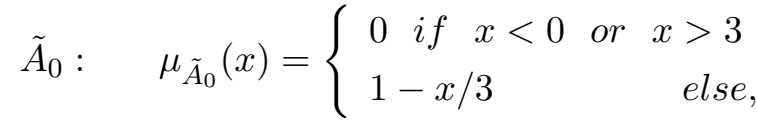

$$
\begin{aligned}
& \tilde{A}_{1}: \quad \mu_{\tilde{A}_{1}}(x)=\left\{\begin{array}{l}
0 \text { if } x<0 \text { or } x>3 \\
x / 3 \text { if } x \geq 0 \text { and } x \leq 3,
\end{array}\right. \\
& \tilde{A}_{n+1}=\tilde{A}_{n-1} \cap \neg \tilde{A}_{n} .
\end{aligned}
$$

The first two sets in the sequence are shown in Fig.5. I start by drawing the graphs of the membership functions of the first two sets in the sequence (see Figure 5.) From $\tilde{A}_{n+1}=$ $\tilde{A}_{n-1} \cap \neg \tilde{A}_{n}$, derive $\tilde{A}_{2}=\tilde{A}_{0} \cap \neg \tilde{A}_{1}$. Notice that on the interval $[0,3], \neg \tilde{A}_{1}=\tilde{A}_{0}, \neg \tilde{A}_{0}=\tilde{A}_{1}$. Moreover, for $x<0$ and for $x>3, \mu_{\tilde{A}_{0} \cap \neg \tilde{A}_{0}}(x)=0, \mu_{\tilde{A}_{1} \cap \neg \tilde{A}_{1}}(x)=0$ and $\mu_{\tilde{A}_{0} \cap \neg \tilde{A}_{1}}(x)=0$. Therefore, $\tilde{A}_{2}=\tilde{A}_{0} \cap \tilde{A}_{0}=\tilde{A}_{0} \cdot \diamond$

Next, write the expression of the membership functions of $\tilde{A}_{2}$ and $\tilde{A}_{3}$ like

$$
\tilde{A}_{2}: \mu_{\tilde{A}_{2}}(x)=\left\{\begin{array}{l}
0 \text { if } x<0 \text { or } x>3 \\
1-x / 3 \text { if } x \geq 0 \text { and } x \leq 3
\end{array}\right.
$$




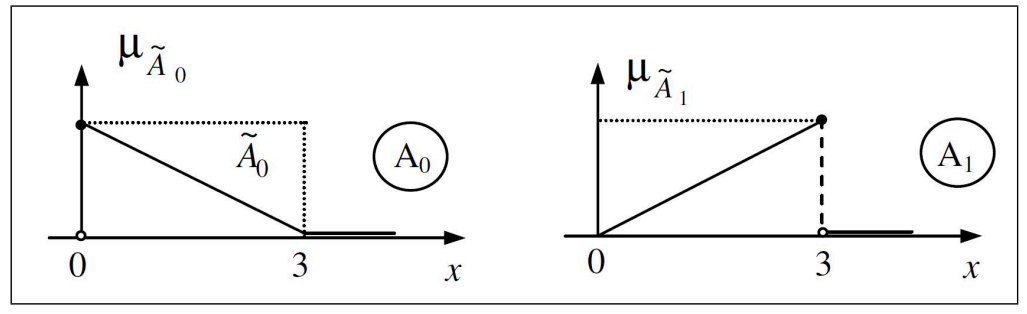

Figure 5: Explanatory graphs.

also, $\tilde{A}_{3}=\tilde{A}_{1} \cap \neg \tilde{A}_{2}, \tilde{A}_{3}=\tilde{A}_{1}$, where

$$
\tilde{A}_{3}:\left\{\begin{array}{l}
0 \text { if } x<0 \text { or } x>3 \\
x / 3 \text { if } x \geq 0 \text { and } x \leq 3
\end{array}\right.
$$

There is no limit for this sequence. Indeed, $\tilde{A}_{n}=\tilde{A}_{n-2} \forall n$. Concluding, this sequence of fuzzy sets has the form $\tilde{A}_{0}, \tilde{A}_{1}, \tilde{A}_{0}, \tilde{A}_{1}, \ldots$, that is, it oscillates with period 2.

\subsection{Fuzzification-defuzzification-based sequences}

Consider the set of fuzzy sets $\Phi=\left\{\tilde{A} \mid \mu_{A}: \mathbf{R} \rightarrow[0,1]\right\}$. Define an operation $\circ: \mathbf{R} \times \Phi \times \Phi \rightarrow \Phi$, where $\mathbf{R}$ is the set of real numbers. An example of such operation is the fuzzification followed by an inference (applying rules and truncation). The operation $\circ$ is defined by $(x \in R,(\tilde{A}, \tilde{B}) \in$ $\Phi \times \Phi) \rightarrow \Phi$, the result being the fuzzy set with the membership function $\mu(u ;(\tilde{A}, \tilde{B}, x))=$ $\min \left(\mu_{\tilde{\tilde{A}}}(x), \mu_{\tilde{B}}(u)\right)$. Then, define a "seed" $x_{0} \in \mathbf{R}$ and the sequence $\tilde{A}_{0}, \tilde{A}_{1}=x_{0} \circ \neg \tilde{A}_{0}, \tilde{A}_{2}=$ $x_{0} \circ \neg \tilde{A}_{1}, \ldots$.

Consider, as above, the sets $\mathbf{R}$ and $\Phi$, and the operation $\circ: \mathbf{R} \times \Phi \rightarrow \Phi$. Moreover, consider a map ô $: \Phi \rightarrow \mathbf{R}$. An example of such a map is the defuzzification, but a more interesting case would be the defuzzification followed by a map from $\mathbf{R}$ to $\mathbf{R}$. Define a "seed" $x_{0} \in \mathbf{R}$, then the sequence $\tilde{A}_{0}, x_{1}=\hat{\mathrm{o}}\left(\mathrm{\circ}\left(x_{0}, \tilde{A}_{0}, \tilde{A}_{0}\right)\right), \tilde{A}_{1}=\left(\circ\left(x_{1}, \tilde{A}_{0}, \tilde{A}_{0}\right)\right), x_{2}=\hat{\mathrm{o}}\left(\mathrm{\circ}\left(x_{1}, \tilde{A}_{0}, \tilde{A}_{0}\right)\right), \ldots$ The generation of this sequence relates to the main subject of study in this topic. Indeed, the procedure above corresponds to an "identity (Mamdani-type) fuzzy logic system", that is, a system based on a single rule "If input is $\tilde{A}$, then output is $\tilde{A}$, followed by a defuzzifier, the defuzzified output value being fed back at the input for fuzzification. Both the defuzzification and the feedback loop are included in the same o operation. This amounts to a simplified fuzzy system in a discrete-time feedback loop configuration. As a matter of example, consider a Mamdani-type fuzzy system with a single rule, $\tilde{A} \rightarrow \tilde{A}$, where the graph of the membership function of $\tilde{A}$ is shown in Figure 6 a) or b).

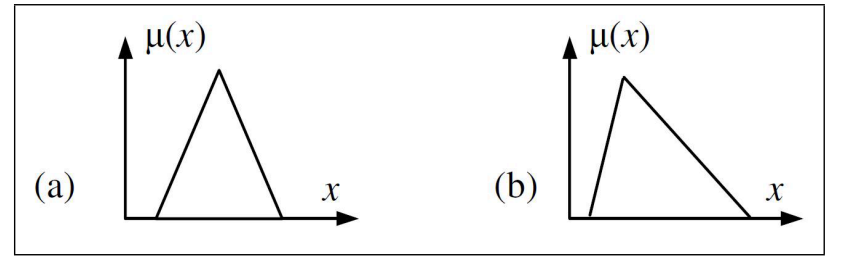

Figure 6: .

For case (a), the sequence $\tilde{A}_{0}, x_{1}=\hat{o}\left(\circ\left(x_{0}, \tilde{A}_{0}, \tilde{A}_{0}\right)\right), \tilde{A}_{1}=\left(\circ\left(x_{1}, \tilde{A}_{0}, \tilde{A}_{0}\right)\right), \quad x_{2}=$ $\hat{o}\left(\circ\left(x_{1}, \tilde{A}_{0}, \tilde{A}_{0}\right)\right), \ldots$ becomes constant $(\tilde{A})$ after two steps, for whatever $x_{0}$ such that $\mu_{\tilde{A}}\left(x_{0}\right)>0$. 
For case (b), there is a limit when $x=\hat{o}(\circ(x, \tilde{A}, \tilde{A}))$ has a solution, that is, the application $\hat{o}(\circ(\cdot, \tilde{A}, \tilde{A}))$ has a fixed point.

\section{$3 \quad$ Iterated fuzzy logic systems}

Essentially, a fuzzy logic system without defuzzification operation at the output is a function from a set of fuzzy sets (the "input" fuzzy sets) to another set of fuzzy sets (the "output" fuzzy sets). A FLS is described by:

i) A finite set of "input" fuzzy sets; I denote these sets by $\tilde{A}_{i}, i=1, \ldots, p$.

ii) A finite set of "output" fuzzy sets; I denote these sets by $\tilde{B}_{j}, j=1, \ldots, q$.

iii) An index function $\imath:\{1, \ldots, p\} \rightarrow\{1, \ldots, q\}$.

iv) An actual "input" fuzzy set (equivalently, membership function), denoted by $\tilde{X} ; \tilde{X}$ is the value of the variable of the function represented by the fuzzy logic system.

v) The equations defining the "output" (the fuzzy set value of the function) corresponding to the input are:

$$
\begin{gathered}
w_{j=\imath(i)}=\max _{x} \min \left\lfloor\mu_{\tilde{X}}(x), \mu_{\tilde{A}_{i}}(x)\right\rfloor \\
\mu_{\tilde{Y}}(x)=\max _{j}\left\lfloor\min \left(w_{1}, \mu_{\tilde{B} 1}(x)\right), \ldots,\left(w_{2}, \mu_{\tilde{B} 2}(x)\right), \ldots,\left(w_{q}, \mu_{\tilde{B} q}(x)\right)\right\rfloor .
\end{gathered}
$$

In applications, fuzzy logic systems are described by rules, in the form IF "input" $\tilde{X}$ is $\tilde{A}_{i}$ THEN "output" $\tilde{Y}$ is $\tilde{B}_{j}$.

where $\tilde{A}_{i}$ corresponds to an input fuzzy set and $\tilde{B}_{j}$ to an output fuzzy set, according to the rule. In principle, nothing prevents that two rules, with the same antecedent have different consequents. Also, rules in the form:

$I F$ "input" $\tilde{X}$ is $\tilde{A}$ THEN "output" $Y$ is $\tilde{B}$ AND $\tilde{Y}$ is $\tilde{B} \prime$;

are allowed. However, in the discussion in this section, I do not include such cases. With the above definition, introduce iterated fuzzy systems as follows:

i) Assume the sets of the input and output fuzzy sets are defined on the same universe of discourse. This condition allows us to perform the iteration. While it is not required, we may require that the input fuzzy sets are identical to the output ones; that is, the function represented by the fuzzy logic system maps a set of fuzzy sets into itself.

ii) At time moment "0", present the fuzzy set $\tilde{X}[0]$ at the input of the fuzzy system.

iii) Assign to the actual input fuzzy set $\tilde{X}[n]$, which is presented at the input of the fuzzy system at the time moment $n$, the output at time moment n-1, $\tilde{Y}[n-1]$, for $n>0$.

This procedure produces the repeated iteration $\varphi^{n}$ of the mapping represented by the fuzzy system $\varphi: M_{U} \rightarrow M_{U}$, where $M_{U}$ is the set of fuzzy sets on the universe of discourse $U$.

In what follows, I present some examples related to typical applications of FLSs. I first address the general case of Sugeno iterated system; next, I address an example involving Sugeno systems; finally, I address an example involving an iterated Mamdani system.

Consider a directed graph $\mathrm{G}=(\mathrm{V}, \mathrm{E})$, where $V$ is the set of vertices and $E$ is the set of directed edges. The graph can be interpreted as the pictorial description (graph) of the index function used in the definition of the input-output mapping represented by the FLS. When the input membership functions differ from the output membership functions, the graph is bipartite. Intuition may induce us to believe that the iteration of a FLS system can have a fixed point only if the graph has a node with a self-loop. This is not true. Even FLSs with bipartite graphs may have a fixed point.

Iterated fuzzy logic systems with singleton-type output. General case. 


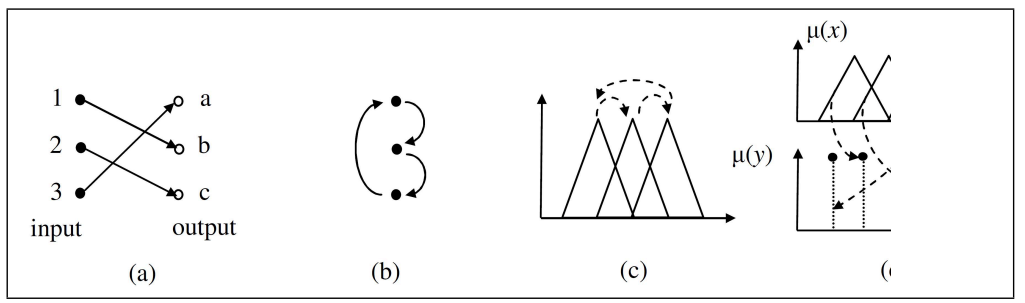

Figure 7: (a) Bipartite graph and loop for a fuzzy logic system with the index functions $1 \rightarrow$ $b, 2 \rightarrow c, 3 \rightarrow a$. (b) respectively $1 \rightarrow 2,2 \rightarrow 3,3 \rightarrow 1$. (c) Example of membership functions for the case (b). Arrows indicate the input to output correspondence. Mamdani-type system. (d) Sugeno-type system.

Consider the set of membership functions $M_{R}=\{\mu: \mathbf{R} \rightarrow[0,1]\}$ and the set of weighted singletons on $\mathbf{R}$, denoted by $S_{R}=\{(\beta, w) \mid \beta \in \mathbf{R}, w \in[0,1]\}$. In $(\beta, w), \beta$ are the values where the singletons occur, that is, $x \neq \beta \Rightarrow \mu_{\beta}(x)=0$, and $\mathrm{w} \in[0,1]$ are the corresponding weights (truth-values), that is $x=\beta \Rightarrow \mu_{\beta}(x)=w$.

Subsequently, formalize the definition of fuzzy logic systems with singleton-type output, which is similar to the classic 0-order Sugeno system, but "deprived" of the defuzzification of the output. Such a system always produces at the output a set of singletons. Thus, the system is a fuzzyinput, fuzzy-output system. When iterated, such a system may have as fixed point a set of singletons that, when presented at the input of the system, produces the same set of singletons at the output.

First, define:

i) A finite set of "input" fuzzy sets; denote these sets by $\tilde{A}_{i}, i=1, \ldots, p$.

ii) A finite set of "output" singleton-type fuzzy sets; denote these sets by $\left(B_{j}, w_{j}=1\right), j=$ $1, \ldots, q$.

iii) An index function $\imath:\{1, \ldots, p\} \rightarrow\{1, \ldots, q\}$.

vi) An actual "input" fuzzy set (equivalently, membership function), denoted by $\tilde{X} ; \tilde{X}$ is the value of the variable of the function represented by the fuzzy logic system.

iv) The equation defining the truncation of the "output" singletons corresponding to the input $\tilde{X}$ is $w_{j=\imath(i)}(\tilde{X})=\max _{x} \min \left\lfloor\mu_{\tilde{X}}(x), \mu_{\tilde{A}_{i}}(x)\right\rfloor$. Here, $w_{j=\imath(i)}(\tilde{X})$ is the truncation value of the singleton with the current number $j$, produced by the rule which for an antecedent $\tilde{A}_{i}$ has consequent $\beta_{j}$.

Notice that the above relation determines the weights of the output singletons, based on the intersection of the fuzzy sets $\tilde{X}$ and $\tilde{A}_{i}$. FLSs as above will be named Sugeno-type.

Proposition. The iteration of a FLS as above defined may have a fixed point.

The proof is based on an example. Consider the FLS defined as in Fig. 8. Assume the membership functions and the rules are:

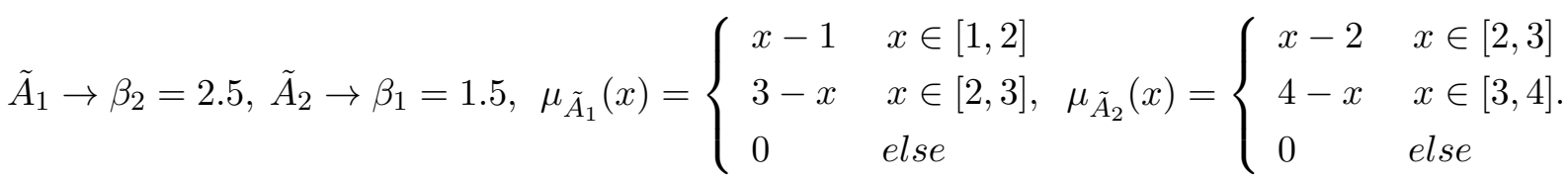

Then, $\tilde{X}$, where $\mu_{\tilde{X}}(x)= \begin{cases}0.5 & x=1.5 \\ 0.5 & x=2.5 \text { is a fixed point of the FLS. } \diamond \\ 0 & \text { else }\end{cases}$

It is not difficult to see that: 


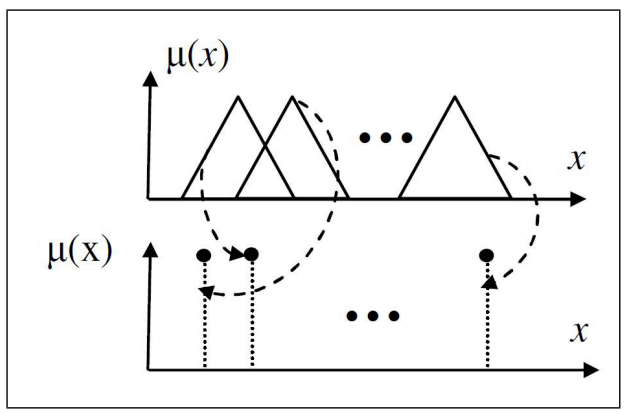

Figure 8: .

Consider a Sugeno-type FLS as above, with continuous input membership functions and with two rules $\tilde{A}_{i} \rightarrow \beta_{j} \tilde{A}_{i+1} \rightarrow \beta_{k}$ where $\mu_{\tilde{A}_{i}}\left(\beta_{j}\right) \neq 0, \mu_{\tilde{A}_{i+1}}\left(\beta_{k}\right) \neq 0$ and $\forall$ h such that $h \neq i \& h \neq i+$ $1 \mu_{\tilde{A}_{h}}\left(\beta_{j}\right)=0$ and $\mu_{\tilde{A}_{h}}\left(\beta_{k}\right)=0$. Then, the FLS has the fixed point $\left(\beta_{j}, \mu_{\tilde{A}_{i}}\left(\beta_{j}\right)\right) \cup\left(\beta_{k}, \mu_{\tilde{A}_{i+1}}\left(\beta_{k}\right)\right)$.

The condition of continuity is needed only to prevent undefined values.

Subsequently, I consider Mamdani-type FLS, that is, systems having "complete" (not singletons) fuzzy sets at the input and the output. Assume that the input and the output membership functions are the same and are isosceles triangular, overlapping two by two (typical case in applications, see figures 7,8 ). Also assume that every rule has a single consequent, that is rules have the form $\tilde{A}_{i} \rightarrow \tilde{A}_{j}$, while rules like $\tilde{A}_{i} \rightarrow \tilde{A}_{j} \vee \tilde{A}_{k}$ are not allowed. Also assume the output of the FLS is defuzzified by the center of gravity (c.o.g.) method. Then,

Proposition. If there is a cycle in the graph of the above described FLS, then there is an initial state of the system that produces an oscillatory iteration.

Indeed, there is an input real value where only one input membership function is non-null and thus selects a single output membership function. Defuzzification of the output by c.o.g. produces a real value; by the assumed conditions, for that value only one input membership function is non-null. Continuing, the cycle in the graph is thus completed. $\diamond$

As a last case, I deal with Sugeno-type FLS. The equation defining the output fuzzy (overall) set (the fuzzy set value of the function represented by the fuzzy system so defined) is:

$$
\mu_{Y}(y, \tilde{X})=\max _{j}\left[\operatorname { m i n } \left(w_{1}(\tilde{X}), \mu_{\beta 1}(y), \ldots,\left(w_{j}(\tilde{X}), \mu_{\beta j}(y), \ldots,\left(w_{q}(\tilde{X}), \mu_{\beta q}(y)\right]\right.\right.\right.
$$

Notice that the last relation represents the union of the weighted output singletons.

The iteration is obtained by imposing $\mu_{X}[n](x)=\mu_{Y}[n-1](y)$. For allowing the iteration, the fuzzy system must satisfy the condition that the input and output membership functions must be the defined and take values on the same space. It is also apparent that, whatever the initial input membership function is, after the first iteration, the input membership function can be but a union of singletons. Consequently, with no loss of generality, I assume that the initial input in the iteration is a set of singletons with the same positions as the output singletons, $\left(\beta_{j}, w_{j}\right), \quad \mathrm{j}=1, \ldots, \mathrm{q}$. These singletons determine a set of $\mathrm{p} \times \mathrm{q}$ values of the input membership functions, $\mu_{j}\left(\beta_{j}\right)$, some of them possibly null.

Therefore, at iteration $n>1, \mu_{i}\left(\beta_{j}[n-1]\right)$. The input truncation values become $\max \left(\mu_{i}\left(\beta_{j}[n-1]\right), w_{j}[n-1]\right)$. Thus, the new values of (truth-degree of) the singletons are:

$$
w_{h}[n]=\max _{j \text { s.t.h=l(j) }} \max _{i}\left(\mu_{i}\left(\beta_{j}[n-1]\right), w_{j}[n-1]\right) ;
$$

The fixed point condition becomes:

$$
w_{h}=\max _{j . t . h=i(j)} \max _{i}\left(\mu_{i}\left(\beta_{j}\right), w_{j}\right)
$$


because the equality must hold for whatever time moment is considered at the two sides. The p-period condition is obtained by requesting that

$$
w_{h}[n]=\max _{j \text { s.t.h } h(j)} \max _{i}\left(\mu_{i}\left(\beta_{j}[n-p-1], w_{j}[n-p-1]\right) .\right.
$$

Example. In this example I deal with a fuzzy logic system as above, with two fuzzy sets defining the input and two singletons defining the output. Consider the two input functions

$$
\begin{gathered}
\mu_{A 1}(x)= \begin{cases}4 x(1-x) & \text { if } x \in[0,1] \\
0 & \text { elsewhere },\end{cases} \\
\mu_{A 2}(x)= \begin{cases}3 x & \text { if } x \in[0,1 / 3] \\
1-(3 / 2)(x-1 / 3) & \text { if } x \in[1 / 3,1] . \\
0 & \text { elsewhere }\end{cases}
\end{gathered}
$$

The index function is described by $1 \rightarrow 2,2 \rightarrow 1$. The output singletons are at $\beta_{1}=0.27$ and $\beta_{2}=0.6$ (see Figure 9).

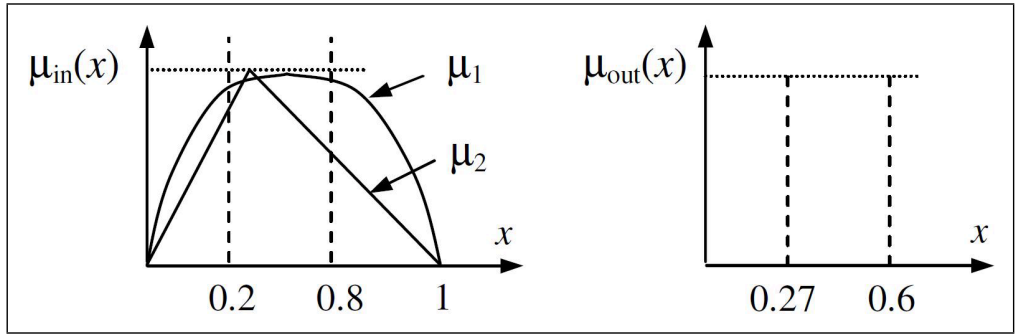

Figure 9: Explanatory graphs for the iteration.

The initial input is $\mu_{X}(0)=\left\{\begin{array}{l}1 \text { if } x=0.2 \\ 1 \text { if } x=0.8 \\ 0 \quad \text { elsewhere }\end{array}\right.$

The first value of the output is $\left(0.27, w_{1}[0]\right)\left(0.6, w_{2}[0]\right)$ where:

$w_{1}[0]=\max \left[\min \left(\mu_{A 2}(0.2), 1\right), \min \left(\mu_{A 2}(0.8), 1\right)\right]=\max (0.6,1-.7)=0.6$, $w_{2}[0]=\max \left[\min \left(\mu_{A 1}(0.2), 1\right), \min \left(\mu_{A 1}(0.8), 1\right)\right]=\max (0.64,0.64)=0.64$.

The output becomes the new value of the variable of the function represented by the fuzzy system. The second value of the output is $\left(\beta_{1}=0.27, w_{1}[1]=0.6\right) \cup\left(\beta_{2}=0.6, w_{2}[1]=0.64\right)$ where:

$$
\begin{aligned}
& w_{1}[1]=\max \left[\min \left(\mu_{A 2}(0.27), w_{1}[0]\right), \min \left(\mu_{A 2}(0.6), w_{2}[0]\right)\right]= \\
& \max [\min (0.81,0.6), \min (0.6,0.64)]=\max (0.6,0.6)=0.6, \\
& w_{2}[1]=\max \left[\min \left(\mu_{A 1}(0.27), w_{1}[0]\right), \min \left(\mu_{A 1}(0.6), w_{2}[0]\right)\right]= \\
& \max (0.64,0.64)=0.64 .
\end{aligned}
$$

Therefore, the output for the second iteration is identical to the output of the first, $\left(\beta_{1}=\right.$ $\left.0.27, w_{1}[1]=0.6\right) \cup\left(\beta_{2}=0.6, w_{2}[1]=0.64\right)$; thus, the iteration result is constant. The result is a fixed point of the system. 


\section{Conclusions}

In this paper, I focused on preparatory topics, namely fuzzy sequences, the iteration of FLS, which are functions defined on sets of (membership) functions, and on their fixed points and their periodicity. Whatever narrow is this topic, it is central in the theory of dynamic fuzzy systems, because both fuzzy logic systems without defuzzifier, and fuzzy algebraic systems apply sets of functions to sets of functions, while fuzzy logic systems with defuzzifier apply sets of functions to real numbers.

The iteration may be convergent (either in a specified metric, or uniformly in the space of the output, that is in the space of the membership functions values) to a limit fuzzy set, or may be periodical, as it has been illustrated using simple examples. More complex behaviors can appear when the output of the FLS is defuzzified. I primarily dealt with fuzzy systems without defuzzifier at the output. The systems with defuzzified output will be dealt with in subsequent papers.

Acknowledgment. I thank several colleagues and the referees for their comments and suggestions. The paper was not supported by, but is related to research performed in the frame of the Romanian Academy.

\section{Bibliography}

[1] P.E. Kloeden, Chaotic iterations of fuzzy sets. Fuzzy Sets and Systems 42 (1991) Volume 42, Issue 1, 5 July 1991, Pages 37-42

[2] H.N. Teodorescu: Fuzzy oscillators. In: H.N. Teodorescu (Editor): Fuzzy Signals and Systems (Proc. AMSE Int. Symposium on Fuzzy Signals and Systems, Cetinje, Yugoslavia, 3-5 Sept. 1990). AMSE Press, France, 1990

[3] H.N. Teodorescu: Controlled Fuzzy Oscillators. in vol: Engineering (Editors: R. Lowen, M. Roubens). Proc 4th IFSA Congress, 1991, Brussels, pp. 211-214

[4] H.N. Teodorescu: Chaotic fuzzy systems: a survey. In: H.N. Teodorescu (Ed): Fuzzy Systems and Signals, vol. 3 (Proc. AMSE Int. Symp. Fuzzy Signals and Systems, Warsaw, June 11-13, 1991). AMSE Press (France)

[5] H.N. Teodorescu: Chaos in fuzzy systems and signals. Vol. Proceedings of the 2nd Int. Conf. on Fuzzy Logic and Neural Networks. Vol. 1., pp. 21-50 (Jono Printing Co., 1992, Iizuka, Japan)

[6] H.N. Teodorescu: Verhulst-type fuzzy growth processes and chaos. Vol. Fuzzy Systems. Proc. ISKIT'92, Iizuka, 1992. pp. 21-28

[7] T. Yamakawa, H.N. Teodorescu, E. Sofron, S. Pavel: Fuzzy models of mechanical phenomena exhibit chaotic behavior. Vol. Fuzzy Systems. Proc. ISKIT'92, Iizuka, 1992. pp. 29-32

[8] H.N. Teodorescu, T. Yamakawa, F. Grigoras, S. Pavel: A hardware implementation of a chaotic fuzzy logic system. Vol. Fuzzy Systems. Proc. ISKIT'92, Iizuka, 1992. pp. 33-38

[9] H.N. Teodorescu, T. Yamakawa, V. Belous, St. Suceveanu: Interpretation of neuro-fuzzy systems in models in management and creativity. Chaos generation. Fuzzy Economic Review. Nov. 1995 (No. 1), pp. 25-42

[10] S. Tamilselvan, K. Vairamanickam, K. Chandrasekhara Rao, Generalized Fuzzy Sequence Spaces. Int. Journal of Math. Analysis, Vol. 4, 2010, no. 45, 2235 - 2242 
[11] B.C. Tripathy and A. J. Dutta, On fuzzy real-valued double sequence spaces, Soochow J. of Mathematics, Volume 32, No. 4, pp. 509-520, October 2006

[12] B.C. Tripathy and A. J. Dutta, On fuzzy real-valued double sequence space. Mathematical and Computer Modelling 46 (2007) 1294-1299

[13] K. Chandrasekhara Rao, V. Karunakaran and K. Vairamanickam, Some Theorems on Fuzzy Matrix Transformation, Int. J. of Math. Analysis, Vol. 4, 2010, no. 5, 243 - 248

[14] B.C. Tripathy, A.J. Dutta, Bounded variation double sequence space of fuzzy real numbers. Computers and Mathematics with Applications. Vol. 59, Issue 2, Jan. 2010, pp. 1031-1037

[15] B.C. Tripathy, B. Sarma, Statistically Convergent Difference Double Sequence Spaces. Acta Mathematica Sinica, English Series, May, 2008, Vol. 24, No. 5, pp. 737-742 (Springer-Verlag 2008)

[16] B.C. Tripathy, Sabita Mahanta, On a class of difference sequences related to the space defined by Orlicz functions. Math. Slovaca 57 (2007), No. 2, 171-178

[17] M. Et, A. Gökhan, and H. Altinok. On statistical convergence of vector-valued sequences associated with multiplier sequences. Ukrainian Mathematical Journal, Vol. 58, No. 1, 2006

[18] L. Cheng, G. Lin, Y. Lan and H. Liu, Measure theory of statistical convergence. Science in China Series A: Mathematics, Vol. 51, Number 12, 2285-2303, DOI: 10.1007/s11425-0080017-z

[19] N. Subramanian, M. Basarir, The Orlicz Space of Entire Sequence of Fuzzy Numbers. Tamsui Oxford J. of Mathematical Sciences 24(1) (2008) 109-122

[20] D. Li, A. Laurent, and P. Poncelet, Discovery of Unexpected Fuzzy Recurrence Behaviors in Sequence Databases. Int. J. of Computer Information Systems and Industrial Management Applications (IJCISIM). Vol. 2 (2010), pp. 279-288

[21] Zhi-Qiang Liu, Leonard T. Bruton, James C. Bezdek, Fellow, IEEE, James M. Keller, Sandy Dance, Norman R. Bartley, and Cishen Zhang, Dynamic Image Sequence Analysis Using Fuzzy Measures. IEEE Transactions on Systems, Man, and Cybernetics-Part B: Cybernetics, Vol. 31, No. 4, August 2001557

[22] M. Gholizadeh, M. M. Pedram, J. Shanbehzadeh, Fuzzy Sequence Mining for Similar Mental Concepts. Proc. Int. Multi-Conf. IMECS 2011, March 16-18, Hong-Kong, pp. 362-367

[23] Bill C.H. Chang, Saman K. Halgamuge, Approximate symbolic pattern matching for protein sequence data. International Journal of Approximate Reasoning 32 (2003) 171-186

[24] J.-Y. Dieulot, P. Borne, Inverse Fuzzy Sum-product Composition and its Application to Fuzzy Linguistic Modelling. Studies in Informatics and Control, Vol. 14, No. 2, 2005, pp. 73-78 